\title{
Designing a Context-Aware Assistive Infrastructure for Elderly Care
}

\author{
Simon Klakegg \\ University of Oulu \\ Oulu, Finland \\ simon.klakegg@oulu.fi \\ Niels van Berkel \\ The University of Melbourne \\ Melbourne, Australia \\ n.vanberkel@student.unimelb.edu.au

\section{Aku Visuri} \\ Hanna-Leena Huttunen \\ Simo Hosio \\ University of Oulu \\ Oulu, Finland \\ aku.visuri@oulu.fi \\ hanna-leena.huttunen@oulu.fi \\ simo.hosio@oulu.fi \\ Permission to make digital or hard copies of all or part of this work for \\ personal or classroom use is granted without fee provided that copies are \\ not made or distributed for profit or commercial advantage and that \\ copies bear this notice and the full citation on the first page. Copyrights \\ for components of this work owned by others than the author(s) must be \\ honored. Abstracting with credit is permitted. To copy otherwise, or \\ republish, to post on servers or to redistribute to lists, requires prior \\ specific permission and/or a fee. Request permissions from \\ Permissions@acm.org. \\ UbiComp/ISWC'17 Adjunct, September 11-15, 2017, Maui, HI, USA \\ (c) 2017 Copyright is held by the owner/author(s). Publication rights \\ licensed to ACM. ACM 978-1-4503-5190-4/17/09...\$15.00 \\ https://doi.org/10.1145/3123024.3124403.
}

\begin{abstract}
We present an assistive healthcare platform, CARE, which aims to provide daily support for elderly caregivers with context-aware, unobtrusive, and actionable information. This information is collected through a plethora of IoT sensors installed strategically at an elderly care centre and is accessed through an Android tablet application. The application's goal is to empower nurses with a better understanding of elderly needs and ultimately, improve the care service. We investigate how IoT devices and sensors can enable a pervasive healthcare system, and discuss a wide-range of important parameters for integration of elderly care practices.
\end{abstract}

\section{Author Keywords}

Elderly Care; Nursing; Assistive Technology; Sensor Infrastructure; System Design.

\section{ACM Classification Keywords}

H.5.m. Information interfaces and presentation (e.g., $\mathrm{HCI}$ ): Miscellaneous;

\section{Introduction}

A pressing concern for global healthcare services is the rapidly aging population. People aged 60 years and older are now the fastest growing group [8], resulting in additional healthcare needs. The need for elderly 


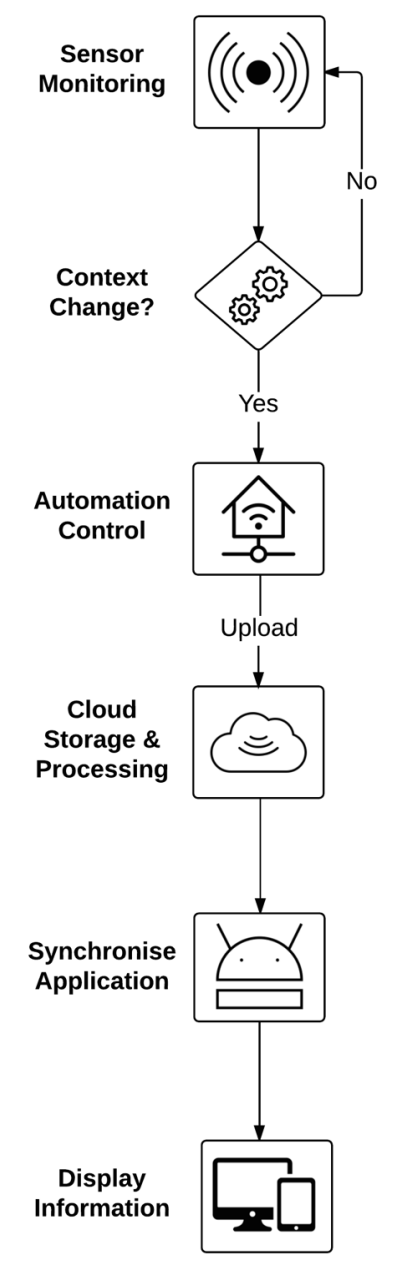

Figure 1: Overview of system components. primary care services is expected to be significant in the near future [3]. Elderly care has suffered from high turnover rates, resulting in a declining quality of care [12]. Noteworthy, this quality decline is directly linked to occupational burnout of care personnel due to high workload and stress [4]. Information and communications technology (ICT) is a valuable tool to improve healthcare personnel effectiveness [11]. Consequently, ICT can help enhance elderly care [6], and reduce operational healthcare costs [1].

\section{Related Work}

Tentori et al. [11] evaluated a mobile and wearable activity monitor, that measures patients' behavioural patterns. Nurses are notified on relevant information through a cellphone and a wearable (armband). Their findings suggest that such systems can improve health care services by increasing efficiency, reducing errors, and raising awareness to patients' needs. Furthermore, the system was generally perceived as useful. It is possible to infer individual personality traits and measure the performance of a group through sensors [9], e.g., a study conducted at a hospital in Boston collected data on physical activity, speech activity, face-to-face interaction, and physical proximity. The researchers were able to estimate the daily average duration of a patients' stay and number of delays using machine learning. Bravo et al. [2] developed an information retrieval system using mobile phones and NFC technology. Information on a patient's prescriptions and health status is collected by touching a patient's tag with a phone. Reducing the time spent on information management enables the nurses to focus on the patients, which in turn increases the care quality. Chu et al. [7] developed a data hiding method for smart devices, which enabled them to hide and encrypt sensitive sensor data, e.g., medical data, into other sensor carrier streams. This enables them to act as automation controllers for sensitive sensor data.

\section{System Design}

The main objective for the system is to provide relevant information to the nurses, assisting them in making better informed decisions throughout their work day. This potentially encourages efficient use of available technical resources and sensors and ultimately improves the quality of care for the patients. The system, CARE [5], is organised into five distinct components, as seen in Figure 1. The sensor infrastructure (devices 1-3 in Table 1), is responsible for capturing fine-grained everyday data on changes in the context (e.g., activity of user, ambient room temperature). Data is transmitted to automation control units (device 4 or 5 in Table 1), and offloaded to the cloud for further processing. The server processes the data, depending on the source (e.g., raw sensors data) before it is stored. This reduces storage requirements for CARE. A custom designed Android application runs on miniature Android PC's (e.g., Remix Mini) and tablets. The application provides the nurses with actionable information regarding the (patient's) context in the care centre. The information is displayed on the tablets, monitors in patients' room, and a TV in the lounge area during daily meetings. We scaled CARE to the size of a collaborating care centre which is approximately 440 square meters, and consists of 14 patient rooms, two hallways, a public lounge and kitchen area. Figure 2 displays an overview of the installation points marked with letters corresponding to the description. 


\begin{tabular}{|c|c|}
\hline Device & Details \\
\hline ThingseePOD & $\begin{array}{l}\text { Location, } \\
\text { humidity, }>100 \\
\text { range, } 2 \text { years } \\
\text { battery }\end{array}$ \\
\hline $\begin{array}{l}\text { Estimote } \\
\text { Sticker }\end{array}$ & $\begin{array}{l}\text { RSSI, } \\
\text { accelerometer, } \\
7 \mathrm{~m} \text { range, } 1 \text { year } \\
\text { battery }\end{array}$ \\
\hline $\begin{array}{l}\text { Estimote } \\
\text { Location } \\
\text { Beacon }\end{array}$ & $\begin{array}{l}\text { Temperature, } \\
\text { ambient light, } \\
70 \mathrm{~m} \text { range, } 5 \text { year } \\
\text { battery }\end{array}$ \\
\hline Remix Mini & $\begin{array}{l}\text { BLE, HDMI, Wi- } \\
\text { Fi, USB 2.0, } \\
\text { Android OS }\end{array}$ \\
\hline Raspberry Pi 3 & $\begin{array}{l}\text { Wi-Fi, BLE, USB } \\
\text { 2.0, HDMI, Linux } \\
\text { OS }\end{array}$ \\
\hline $\begin{array}{l}\text { Google } \\
\text { Chromecast }\end{array}$ & $\begin{array}{l}\text { HDMI, Wi-Fi, } \\
\text { Android OS }\end{array}$ \\
\hline 19” Monitor & HDMI, Dell \\
\hline Tablet & $\begin{array}{l}\text { Wi-Fi, BLE, } \\
\text { Android OS, } 3 \\
\text { day battery }\end{array}$ \\
\hline
\end{tabular}

Table 1: Overview of system hardware.

\section{A. Bed Sensor}

Each bed is equipped with an Estimote Sticker ${ }^{1}$. This is a small (3 $\mathrm{mm}$ thin) and lightweight BLE (Bluetooth Low Energy) beacon which broadcasts contextual data (e.g., orientation, accelerometer) using the Nearable protocol [10]. As the patient enters or exits the bed, the event will be measured by the sticker and the data will be communicated to nearby devices. This sensor enables nurses to infer whether a patient is in bed, whether they have fallen out of bed, and number of hours slept.

\section{B. Chromecast}

A Chromecast is connected to the TV in the main lounge. This enables the nurses to view patient data from the application on a large monitor during

\section{handover meetings.}

\section{Raspberry Pi 3}

A Raspberry Pi 3 act as an automation controller for the ThingseePODs. It receives the sensor data from the Thingsee Wirepas [13] mesh network and transfers it to our database.

\section{Nurse Sticker}

To monitor the nurses' workflow and interactions with patients, we equip them with Estimote Stickers. As this beacon actively broadcasts in a small proximity, we can infer the location of the nurses throughout the facility. The broadcast is detected by the present Remix Minis, and proximity is deducted based on the RSSI values. Based on this data the application can notify the user when a patient has not been in proximity of a nurse for an extended period (i.e., there has been no check-up).

\section{Nurse Tablet (Application)}

The nurses are equipped with Android tablets ( $7 ")$, which runs a custom developed application. The application retrieves the latest processed sensor data from the cloud and presents it in a simple and understandable manner to the user [5].

\section{E. Room Sensor}

The patients living rooms are also equipped with a Estimote Location Beacon. This is a larger and more advanced version (e.g., extra sensors and functionality) of the Estimote Sticker. The device is installed on the wall and measures ambient light and the room temperature. Consequently, it can detect if a patient has forgotten to close their window or turn off the lights during night. This simplifies the nightly room inspections for the nurse.

\section{F. Room Display}

A 19" monitor is mounted in the living room to display relevant information (e.g., status, hours of sleep tonight, time since last shower, number of check-ups today) regarding the patients when the nurses are near.

\section{G. Shower Sensor}

A rugged and waterproof IoT device named ThingseePOD ${ }^{2}$ is installed in each patient's bathroom. The unit has a range of built in sensors and communicates with other PODs using Wirepas Connectivity [13], enabling an adaptive multi-hop mesh network topology. We utilise the humidity sensor to detect when patients shower. As a result, the nurses no longer have to keep track of this task and will be reminded when the task needs to be completed. 


\section{H. Remix Mini}

We install multiple Remix $\mathrm{Mini}^{3}$ at the care centre to receive BLE broadcasts from the beacons. The Android computer act as an automation controller and receives data from both the infrastructure and personnel. One Remix Mini is also connected to the living room monitor and is responsible for presenting information.

\section{Toilet Sensor}

In order to detect when a patient goes to the toilet, an Estimote Sticker is attached to the actuator. It will detect the accelerometer changes when the button is

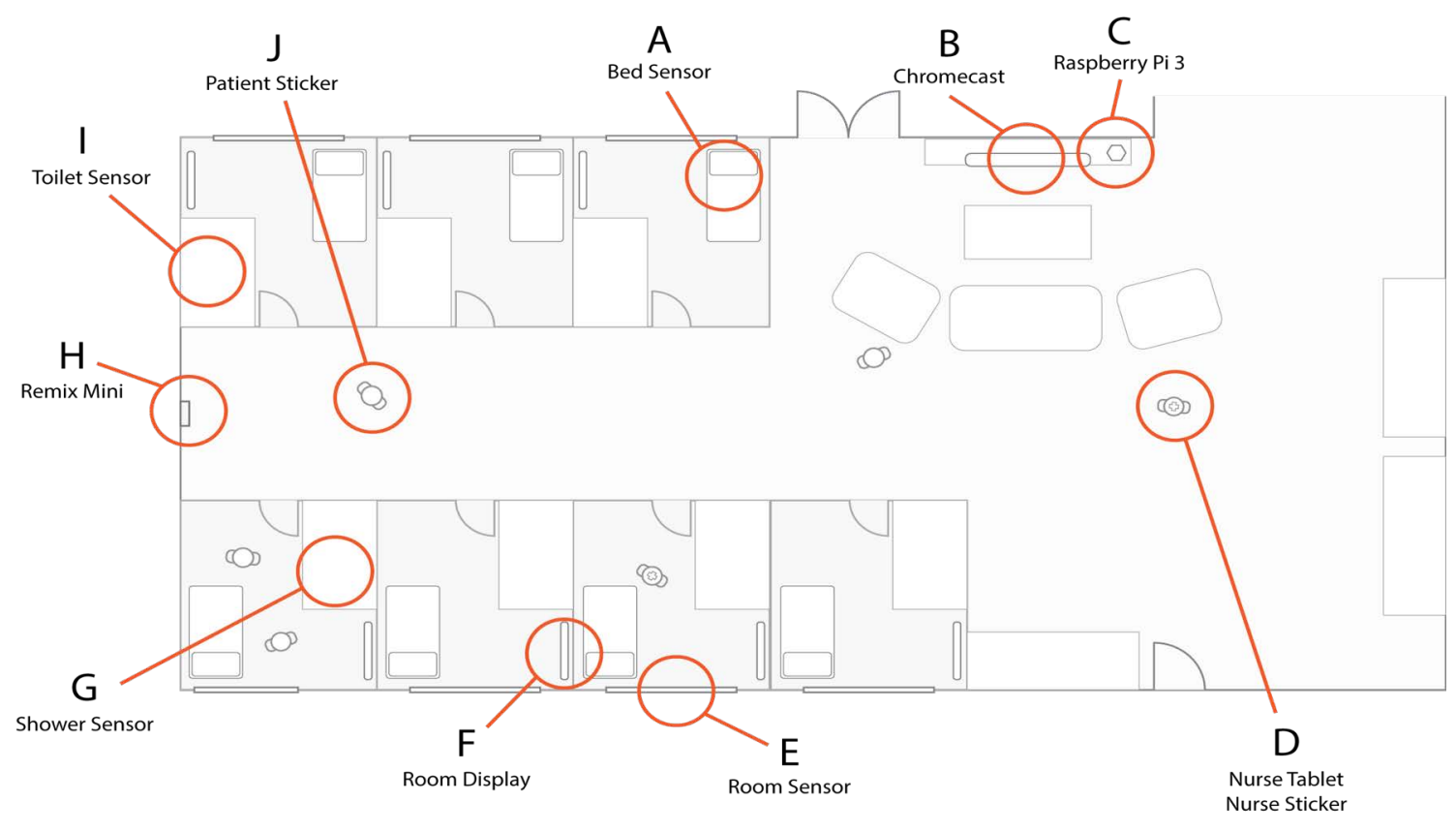

Figure 2. Floorplan of the care centre with key installation points marked. pushed. Resultantly, the application can warn nurses when the patient has not visited the toilet for a prolonged period.

\section{J. Patient Sticker}

Similarly to the nurses, we equip patients with Estimote Stickers to monitor their context. This enables the nurses to better understand patients wellbeing, by having an overview of their activity, when they last moved, location, time spent socialising, and activity level through the application.

\footnotetext{
${ }^{3}$ www.jide.com/mini
} 

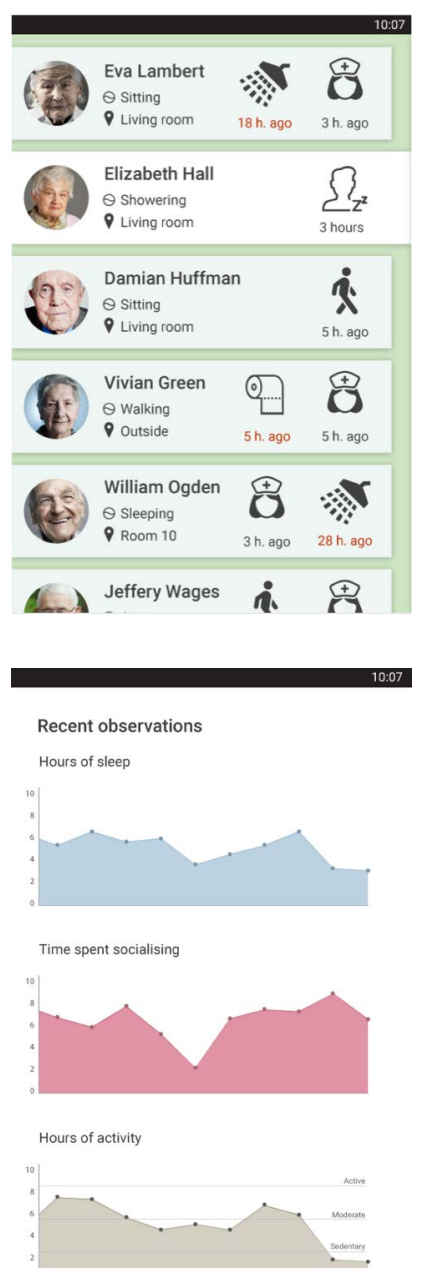

Figure 3: UI of CARE application. Top: Overview of residents' status and care needs. Bottom: Historical overview of sensor data.
We design an Android application that runs on tablets provided to the nurses (Figure 3 ). The top view shows an overview of all the patients residing in the facility and displays: patient's name, picture, current location (approximate), amount of activity (e.g., sedentary, moderate, active), pressing tasks (e.g., 5 h since last check-up), and other important information/notes (e.g., only slept 3 hours tonight). In the bottom view, we show a historical overview of a specific patient for the last week, month and year. This overview is imperative to visually identify potential trends in the patient's behaviour, health, and overall wellbeing. For example, if the patient starts to slowly isolate herself over time, this tendency will be visualised in the 'Time spent socialising' section. The application can therefore raise awareness to patients' needs that would otherwise go unnoticed.

\section{Discussion}

In comparison to previous work, we do rely on wearables and manual input of contextual data [11] or interaction with NFC tags [2]. CARE's infrastructure intends to raise awareness of the residents' needs, ensuring they receive the required care, e.g., if the resident has not moved for a long time (i.e., danger of bedsores) or been checked upon recently, the application can inform the staff. As there are monitors in each of the residents' rooms, their information is displayed when the nurses are nearby. This allows the worker to continue their task at hand, without interacting directly with the system, hands-free. When the nurses are on the move, they can simply bring the small tablet with them. The nurses are never forced to use the application, and it is meant as an optional, but always available tool. It does not require manual input. We consider these as key parameters for a system like
CARE to work and have a significant impact in elderly care practices. The nurses are already overloaded, and adding more to that would be counterintuitive, and unreasonable. Moreover, by measuring nurse-patient interactions and time in care, one can assess care bottlenecks problems and identify future areas of improvement. The management can utilise this data to make strategical decision about the operations of the care home and recruitment needs. We rely on off-theshelf sensors with a very long battery lifetime (over one year) with continuous and predetermined sampling rates. Consequently, the infrastructure requires very little maintenance, can collect data for extended periods and does not interfere with existing tools.

\section{Conclusion}

This paper describes a context-aware assistive infrastructure for elderly care, CARE. CARE monitors context changes and provides sensible information for elderly care staff through a custom designed Android tablet application. This information allows the nurses to make faster and informed decisions, in a continuously challenging and hectic work environment. We argue that by enabling the nurses' awareness of the patient needs, one can help enhance their efficiency, and improve the overall care services. Our initial results are promising. We conducted a proof-of-concept implementation of CARE to ensure that all components work as a whole. However, the focus of this paper is on the requirements elicitation for our sensing

infrastructure, its functionality, and the opportunities it presents. Therefore, it is qualitative in presentation. In a future publication, we shall present a longitudinal evaluation of CARE, its performance and developed algorithms for understanding elderly care environments and elderly needs. 


\section{Acknowledgements}

This work is partially funded by the Academy of Finland (Grants 276786-AWARE, 286386-CPDSS, 285459iSCIENCE, 304925-CARE), the European Commission (Grant 6AIKA-A71143-AKAI), and Marie SkłodowskaCurie Actions (645706-GRAGE).

\section{References}

1. Margrethe Aanesen, Ann Therese Lotherington and Frank Olsen. 2011. Smarter elder care? A costeffectiveness analysis of implementing technology in elder care. Health Informatics Journal, 17 (3). 161-172.

2. J. Bravo, R. Hervas, C. Fuentes, G. Chavira and S. W. Nava. 2008. Tagging for nursing care. In 2008 Second International Conference on Pervasive Computing Technologies for Healthcare, 305-307. 10.1109/PCTHEALTH.2008.4571097

3. Timothy M Dall, Paul D Gallo, Ritasree Chakrabarti, Terry West, April P Semilla and Michael V Storm. 2013. An aging population and growing disease burden will require alarge and specialized health care workforce by 2025. Health Affairs, 32 (11).

4. Maria Engström, Brigitta Ljunggren, Ragny Lindqvist and Marianne Carlsson. 2006. Staff satisfaction with work, perceived quality of care and stress in elderly care: psychometric assessments and associations. Journal of Nursing Management, 14 (4). 318-328.

5. Simon Klakegg, Niels van Berkel, Aku Visuri, Chu Luo, Jorge Goncalves, Simo Hosio, Hanna-Leena Huttunen and Denzil Ferreira. 2017. Informing Caregivers Through an Assistive Tool: An Investigation of Elderly Care Metrics. In Proceedings of the 31st British Human Computer Interaction Conference, BCS Learning and Development Ltd.

6. Rita Kobb, Nannette Hoffman, Robert Lodge and Sheri Kline. 2003. Enhancing elder chronic care through technology and care coordination: report from a pilot. Telemedicine Journal and e-HEALTH, 9 (2). 189-195.

7. Chu Luo, Angelos Fylakis, Juha Partala, Simon Klakegg, Jorge Goncalves, Kaitai Liang, Tapio Seppänen and Vassilis Kostakos. 2016. A data hiding approach for sensitive smartphone data. In Proceedings of the 2016 ACM International Joint Conference on Pervasive and Ubiquitous Computing, ACM, 557-468.

8. United Nations. 2015. 2015 Revision of World Population Prospects. URL:

http://www.un.org/en/development/desa/publicati ons/world-population-prospects-2015-revision.htm (accessed 25 May 2017)

9. D. Olguin Olguin, P. A. Gloor and A. Pentland. 2009. Wearable sensors for pervasive healthcare management. In 2009 3rd International Conference on Pervasive Computing Technologies for Healthcare, 1-4. 10.4108/ICST.PERVASIVEHEALTH2009.6033

10. Witek Socha. 2016. What are Estimote Stickers? URL: https://community.estimote.com/hc/enus/articles/203323543-What-are-EstimoteStickers- (accessed 25 May 2017)

11. M. Tentori and J. Favela. 2008. Monitoring behavioral patterns in hospitals through activityaware computing. In 2008 Second International Conference on Pervasive Computing Technologies for Healthcare, 173-176. 10.1109/PCTHEALTH.2008.4571062

12. Huey-Ming Tzeng and Shaké Ketefian. 2002. The relationship between nurses' job satisfaction and inpatient satisfaction: an exploratory study in a Taiwan teaching hospital. Journal of nursing care quality, 16 (2). 39-49.

13. Wirepas. 2017. Unique technology for device communication. URL: http://www.wirepas.com/ (accessed 25 May 2017) 